\title{
LA SALINIDAD INCREMENTA EL CONTENIDO DE FLAVONOIDES, DE ANTOCIANINAS Y EL POTENCIAL HIPOGLUCEMIANTE DE TOMATILLO (Physalis ixocarpa)
}

\author{
Salinity increases the contents of flavonoids, anthocyanins and the hypoglicemic \\ potential of tomatillo (Physalis ixocarpa)
}

Imelda Rosas-Medina ${ }^{1}$, Aurelio Colmenero-Robles ${ }^{1}$, Néstor Naranjo-Jiménez ${ }^{2}$, José Antonio Ávila-Reyes ${ }^{2}$ y Norma Almaraz-Abarca ${ }^{2 *}$

${ }^{1}$ Instituto Politécnico Nacional, Secretaría de Investigación y Posgrado, Unidad Profesional Adolfo López Mateos, Zacatenco, Ciudad de México, México, 07738. ${ }^{2}$ Instituto Politécnico Nacional, Centro Interdisciplinario de Investigación para el Desarrollo Integral Regional unidad Durango, Sigma 119, Fraccionamiento 20 de Noviembre II, Durango, Durango, México, 34220.

*Autor de correspondencia: nalmaraz@ipn.mx

\section{Resumen}

En el presente estudio se determinó el efecto de la salinidad sobre los niveles de flavonoides y de antocianinas, así como del potencial inhibidor de las enzimas $\alpha$-glucosidasa y $\alpha$-amilasa de frutos de tomatillo (Physalis ixocarpa), una especie con relevante interés alimenticio y comercial de México. Algunos reportes han revelado que las condiciones de estrés ambiental pueden incrementar los niveles y diversificar la composición fenólica, y por lo tanto las propiedades biológicas de algunas especies de plantas. Los resultados revelaron que una concentración de $120 \mathrm{mM}$ de $\mathrm{NaCl}$, adicionada diariamente durante 15 días a plantas de 42 días de edad provocó el incremento significativo de los contenidos de flavonoides y de antocianinas de los frutos de tomatillo con respecto al control, en 1.16 y 1.46 veces, respectivamente. Los resultados también revelaron que los extractos a 200 $\mathrm{mg} / \mathrm{mL}$ de frutos de plantas cultivadas en $120 \mathrm{mM}$ de $\mathrm{NaCl}$ incrementaron su potencial inhibidor de $\alpha$-glucosidasa 2.89 veces más que los extractos, a esa misma concentración, de frutos de las plantas control. Los frutos de tomatillo no mostraron potencial inhibidor de $\alpha$ amilasa, ni este potencial se estimuló con el estrés salino. Frutos crecidos en las diferentes condiciones salinas fueron discriminados por un análisis de componentes principales basado en los contenidos de flavonoides, de antocianinas, y en el potencial inhibidor de $\alpha$-glucosidasa, por lo que estos parámetros pueden representar una herramienta de control de calidad con relación a las condiciones de crecimiento de las plantas de esta especie.

Palabras clave: $\alpha$-glucosidasa, flavonoides, antocianinas.
Abstract
In the current study, the effect of salinity on the levels of flavonoids and anthocyanins was determined, as well as the inhibitory potential of the enzymes $\alpha$ - glucosidase and $\alpha$-amylase of tomatillo fruits (Physalis ixocarpa), a species with relevant nutritional and commercial 
interest from Mexico. Some reports have revealed that environmental stress can increase the levels and diversity of phenolic composition, and therefore the biological properties of some plant species. The results revealed that the concentration of $120 \mathrm{mM}$ of $\mathrm{NaCl}$, added daily for 15 days to 42-day-old plants caused the increase in flavonoid and anthocyanin contents of tomatillo fruits with respect to control, in 1.16 and 1.46 times, respectively. The results also revealed that the extracts at $200 \mathrm{mg} / \mathrm{mL}$ of fruits of plants grown in $120 \mathrm{mM} \mathrm{NaCl}$ increased 2.89 times their capacity to inhibit $\alpha$-glucosidase compared to the extracts of the control plants fruits. Tomatillo fruits showed no potential as $\alpha$ amylase inhibitors, nor was this potential stimulated with saline stress. Fruits grown in different saline conditions were discriminated by a principal component analysis based on the contents of flavonoids and anthocyanins, as well as on the $\alpha$-glucosidase inhibitory potential, so these parameters can represent a quality control tool, in relation to the growth conditions, of this species.

Keywords: $\alpha$-glucosidase, flavonoids, anthocyanins.

\section{Introducción}

La inhibición de la actividad de las enzimas $\alpha$-amilasa y la $\alpha$-glucosidasa puede interferir en la digestión de carbohidratos y disminuir el estado hiperglicémico (Choudhary et al., 2011). Ésta es una estrategia para el tratamiento de la hiperglicemia, que es un estado característico de personas con diabetes (Hadjzadeh et al., 2015). En el organismo humano, la $\alpha$-amilasa degrada carbohidratos complejos como el almidón y el glucógeno a azúcares más simples como $\alpha$-dextrina y los oligosacáridos maltosa y maltotriosa, los cuales después pueden degradarse a glucosa por la acción de la $\alpha$-glucosidasa, incrementando sus niveles en el plasma (García-Luna y López-Gallardo, 2007; Asgar, 2013). Actualmente, existe la tendencia a buscar terapias alternativas basadas en productos naturales, provenientes muchos de ellos de plantas.

México es uno de los países con mayor diversidad vegetal en el mundo, lo que hace que se tenga una gama importante de especies de plantas (Heinrich et al., 2014) que son fuente de una quimiodiversidad todavía mayor. Además de la diversidad química natural encontrada en las plantas, es posible favorecer el aumento de la concentración y ampliar la diversidad de bioproductos por medio de estrategias como el someter a las plantas a condiciones de estrés durante su crecimiento (Martínez-Lüscher et al., 2014; Minh et al., 2016). La salinidad afecta la biosíntesis y acumulación de compuestos bioactivos de plantas (Minh et al., 2016). La flexibilidad de respuestas es una característica importante y esencial para que las plantas puedan sobrevivir en condiciones de estrés. Para ello, diferentes especies han desarrollado mecanismos variables de señales que les permiten reprogramar rápidamente su metabolismo, condición fisiológica, y su desarrollo (Asensi-Fabado et al., 2017).

Dentro del género Physalis, la especie más estudiada desde diferentes puntos de vista ha sido $P$. peruviana, la cual crece de manera natural en América del Sur. Para $P$. peruviana se han reportado propiedades citotóxicas de carcinoma hepático (Hassan et al., 2017), cáncer de próstata, y carcinoma renal $(\mathrm{Xu}$ 
et al., 2017), entre otras. Sin embargo, para Physalis ixocarpa o tomatillo, la cual tiene una importante relevancia culinaria en México y también es rica en bioproductos (González-Mendoza et al., 2010), existe todavía un importante vacío de conocimiento sobre sus propiedades biológicas. Un estudio reveló actividad apoptótica de $P$. ixocarpa en células de cáncer de colon (Choi et al., 2006). El objetivo del presente estudio fue determinar los cambios en los contenidos de flavonoides y antocianinas, así como el potencial inhibidor de $\alpha$-glucosidasa y $\alpha$ amilasa de tomatillo sometido a estrés salino.

\section{Materiales y métodos}

\section{Material vegetal y tratamientos salinos}

Se utilizaron semillas de Physalis ixocarpa variedad Rendidora que fueron proporcionadas en 2014 por productores de Oaxaca y que se han mantenido a través de resiembras en el Centro Interdisciplinario de Investigación para el Desarrollo Integral Regional unidad Durango del Instituto Politécnico Nacional (CIIDIR IPN Durango). Esas semillas se sembraron y las plantas se cultivaron en el invernadero del mismo Centro, de acuerdo a Cobaleda-Velasco et al. (2017) hasta su fructificación. En los tratamientos salinos, cada una de 10 plantas se mantuvieron en contenedores de plástico de $4 \mathrm{~L}$ y se regaron, a partir del día 42 después de la siembra y durante los 15 días siguientes, con $1 \mathrm{~L}$ de una solución $90 \mathrm{mM}$ o $120 \mathrm{mM}$ de $\mathrm{NaCl}$. El inicio de la aplicación de la solución salina correspondió a 15 días antes de la etapa de desarrollo GS3 de la escala $\mathrm{BBCH}$ (Centro de Investigación Biológica de Variedades Vegetales e Industria Química), que corresponde a plantas de 57 días, las cuales han desarrollado mucho follaje y tienen frutos maduros (Ramírez et al., 2013). En el tratamiento control, el riego de un número igual de plantas se hizo con agua corriente. De cada tratamiento, se colectaron 10 frutos maduros (un fruto por planta). Cada tratamiento tuvo tres réplicas.

\section{Preparación de los extractos}

Los frutos de tomatillo de cada tratamiento, independientemente, se secaron combinaron y posteriormente se molieron. Un gramo de muestra seca y molida se maceró en $10 \mathrm{~mL}$ de etanol al $80 \%$ (v/v) en agitación durante 12 horas. Después, se centrifugo a $8000 \mathrm{rpm}$ durante 10 minutos, el sobrenadante se recolecto y la pastilla fue re-extractada bajo las mismas condiciones. Los dos sobrenadantes de una misma muestra se combinaron y se llevaron a sequedad. El extracto seco se resuspendió en etanol al $20 \%(\mathrm{v} / \mathrm{v})$, en el volumen que permitió obtener una concentración de $2 \mathrm{mg} / \mathrm{mL}$. De los extractos, se tomaron alícuotas para realizar los análisis de contenidos de flavonoides y antocianinas, y de la actividad hipoglucemiante.

Determinación del contenido de flavonoides

El contenido de flavonoides se determinó utilizando el método descrito por Ordoñez et al. (2006). El contenido de flavonoides se estimó a partir de una curva de calibración de quercetina $\left(\mathrm{A}_{420}=\right.$ 0.3261 [quercetina] $+0.0277, \mathrm{r}=0.9957$ ) y los resultados se expresaron en microgramos equivalentes de quercetina por gramo de extracto seco ( $\mu \mathrm{g} \mathrm{EQ/g} \mathrm{ES).}$

Determinación de los contenidos de antocianinas 
Los contenidos de antocianinas monoméricas se determinaron con el método de $\mathrm{pH}$ diferencial descrito por Giusti y Wrolstad (2001), usando el peso molecular (449.2) y la absorción molar (26 900) de cianidina-3-O-glucósido como referencia, debido a que esta antocianina es de las más comúnmente encontradas. Los valores se expresaron como miligramos por gramo de extracto $\operatorname{seco}(\mathrm{mg} / \mathrm{g} \mathrm{DE}) .5$

Capacidad inhibidora de $\alpha$-glucosidasa y de $\alpha$-amilasa

La evaluación del potencial hipoglucemiante de los extractos se realizó determinando la capacidad inhibidora de las enzimas $\alpha$-glucosidasa de Bacillus stearothermophilus y $\alpha$ amilasa pancreática porcina. de acuerdo a Kim et al. (2010) y Kim et al. (2011), respectivamente. En ambos casos se usó una solución de acarbosa $(10 \mu \mathrm{g} / \mathrm{mL})$ como referencia. Se estimó el porcentaje de inhibición de diferentes concentraciones de extractos (120 a 200 $\mathrm{mg} / \mathrm{mL}$ ) y la actividad se expresó como la concentración efectiva inhibitoria al $50 \%$ ( $\left.\mathrm{IC}_{50}\right)$ en $\mathrm{mg} / \mathrm{mL}$.

\section{Análisis de datos}

Los datos se sometieron a un ANOVA para evaluar las diferencias entre muestras $(\mathrm{p}<0.05)$ y la discriminación entre medias se estimó con la prueba de Tukey. Los resultados se procesaron con el programa de cómputo XLSTAT 2017.03.44803. Para determinar la contribución de cada uno de los parámetros evaluados para diferenciar las muestras, los datos se sometieron a un análisis de componentes principales (PCA), usando el programa de cómputo Past 3.

\section{Resultados y Discusión}

\section{Contenido de flavonoides y antocianinas}

En el Cuadro 1 se muestran los contenidos de flavonoides y de antocianinas de los frutos de plantas de tomatillo sometidas a estrés salino y de plantas control. Diferencias significativas se pueden apreciar entre los contenidos de ambos compuestos en los frutos provenientes de los diferentes tratamientos, siendo mayor el contenido en los frutos sometidos a la más alta concentración de $\mathrm{NaCl}$ evaluada. Sin embargo, mientras que el contenido de flavonoides disminuyó en la concentración de $90 \mathrm{mM}$ de $\mathrm{NaCl}$, con respecto al control, antes de aumentar a la concentración salina de $120 \mathrm{mM}$, el aumento de la concentración de antocianinas fue progresiva de acuerdo al incremento de la concentración de la solución salina adicionada. $\mathrm{La}$ concentración de flavonoides a la concentración de $120 \mathrm{mM}$ de $\mathrm{NaCl}$ con respecto al control se incrementó en 1.16 veces, mientras que la de antocianinas en 1.46

veces. 
Cuadro 1. Efecto de dos diferentes concentraciones de $\mathrm{NaCl}$, durante 15 días, en el contenido de flavonoides y antocianinas de frutos de tomatillo (Physalis ixocarpa var. Rendidora).

\begin{tabular}{ccc}
\hline Tratamiento & $\begin{array}{c}\text { Flavonoides } \\
(\mathrm{mg} E Q / g \text { ES })\end{array}$ & $\begin{array}{c}\text { Antocianinas } \\
(\mu \mathrm{g} / \mathrm{g} \mathrm{ES})\end{array}$ \\
\hline Control & $1.46 \pm 0.03 \mathrm{~b}$ & $1.50 \pm 0.42 \mathrm{c}$ \\
$90 \mathrm{mM}$ & $0.49 \pm 0.02 \mathrm{c}$ & $1.8 \pm 0.43 \mathrm{~b}$ \\
$120 \mathrm{mM}$ & $1.70 \pm 0.03^{\mathrm{a}}$ & $2.2 \pm 0.34^{\mathrm{a}}$ \\
\hline
\end{tabular}

Letras diferentes dentro de una misma columna representan diferencias significativas $(\mathrm{p}<0.05)$.

EQ: Equivalentes de quercetina; ES: Extracto seco.

Varios autores han reportado que aumentos en el nivel de estrés salino provoca aumentos en la acumulación de flavonoides (Chutipaijit et al., 2009; Minh et al., 2016). Sin embargo, también existen reportes que informan sobre un efecto contrario del estrés salino en la acumulación de estos compuestos (Telesiński et al., 2008). Las diferentes respuestas de las plantas pueden depender de la susceptibilidad de éstas al estrés salino (Walia et al., 2005). Los resultados de la Tabla 1 sugieren que en Physalis ixocarpa la acumulación de flavonoides y antocianinas puede ser un mecanismo químico importante para tolerar condiciones de salinidad.
Capacidad inhibidora de $\alpha$-glucosidasa $y$ de $\alpha$-amilasa

Los valores del porcentaje de inhibición de la actividad de $\alpha$ glucosidasa de diferentes concentraciones de extractos de tomatillo crecido en y sin estrés salino se presentan en el Cuadro 2; en ella se puede apreciar que la capacidad de inhibición de esta enzima incrementó con el incremento de la concentración del extracto, de 120 a $200 \mathrm{mg} / \mathrm{mL}$, en 2.78 veces sin estrés salino y en 12.6 veces en frutos que sufrieron el estrés salino de $120 \mathrm{mM}$ de $\mathrm{NaCl}$. A $200 \mathrm{mg} / \mathrm{mL}$, el potencial inhibidor de $\alpha$-glucosidasa de los frutos de plantas crecidas en $120 \mathrm{mM}$ de $\mathrm{NaCl}$ fue 2.89 veces más alto que el de las plantas control.

Cuadro 2. Inhibición (\%) de la actividad de $\alpha$-glucosidasa de tomatillo (Physalis ixocarpa) sometido a diferentes concentraciones de $\mathrm{NaCl}(\mathrm{mM})$.

\begin{tabular}{cccc}
\hline $\begin{array}{c}\text { Concentración de extracto } \\
(\mathrm{mg} / \mathrm{mL})\end{array}$ & 0 & 90 & 120 \\
\hline 200 & $25.87 \pm 3.36 \mathrm{a}$ & ND & $75.02 \pm 0.071 \mathrm{a}$ \\
180 & $21.14 \pm 3.21 \mathrm{~b}$ & ND & $59.68 \pm 0.070 \mathrm{~b}$ \\
160 & $16.74 \pm 0.04 \mathrm{c}$ & ND & $30.81 \pm 0.09 \mathrm{c}$ \\
140 & $10.58 \pm 0.11 \mathrm{~d}$ & ND & $12.04 \pm 0.074 \mathrm{~d}$ \\
120 & $9.29 \pm 0.12 \mathrm{~d}$ & ND & $5.91 \pm 0.10 \mathrm{e}$ \\
\hline
\end{tabular}

Letras diferentes dentro de una misma columna representan diferencias significativas $(p<0.05)$. ND: No detectada. 
En el Cuadro 3 se muestran los valores de $\mathrm{IC}_{50}$ de la actividad inhibidora de $\alpha$-glucosidasa de los frutos de Physalis ixocarpa, en la que se aprecia el incremento de la actividad (reducción del valor $\mathrm{IC}_{50}$ ) en los tomatillos de plantas crecidas en $120 \mathrm{mM}$ de $\mathrm{NaCl}$. Es sobresaliente el hecho de que el incremento en el potencial inhibidor de la actividad de $\alpha$-glucosidasa de tomatillo sometido a estrés salino fue coincidente principalmente con los cambios de concentración de los flavonoides (Cuadro 1). Sin embargo, la actividad inhibidora de la $\alpha$-glucosidasa, del extracto de los frutos de Physalis ixocarpa sometidos a estrés salino fue más bajo que el potencial de la acarbosa $(7.56 \mathrm{mg} / \mathrm{mL})$. A pesar de ello, el consumo de frutos de tomatillo de plantas crecidas en $120 \mathrm{mM}$ de $\mathrm{NaCl}$ pueden tener un efecto benéfico para el control de la hiperglicemia, ya que la acarbosa, que ha sido utilizada clínicamente para el tratamiento de diabetes (Sun y Miao, 2020), tiene efectos gastrointestinales y hepáticos secundarios (Asgar, 2013; Vinayagam et al., 2016). Los frutos de tomatillo de plantas del tratamiento control no mostraron actividad inhibidora de $\alpha$-amilasa, ni el estrés salino estimuló algún potencial de inhibición de esta enzima.

Cuadro 3. Concentración inhibidora del $50 \%\left(\mathrm{IC}_{50}\right)(\mathrm{mg} / \mathrm{mL})$ de la actividad de $\alpha$ glucosidasa de tomatillo (Physalis ixocarpa) sometidos a diferentes concentraciones de $\mathrm{NaCl}(\mathrm{mM})$.

\begin{tabular}{rccc}
\hline Muestra & 0 & 90 & 120 \\
\hline Tomatillo & $>200$ & ND & $175.65 \pm 0.41$ \\
\hline
\end{tabular}

ND: No detectada.

$P C A$

Los resultados del análisis de PCA, basado en las determinaciones del contenido de flavonoides, de antocianinas, y de $\mathrm{IC}_{50}$ de inhibición de $\alpha$ glucosidasa se muestran en la Figura 1. Dos componentes principales explicaron el $99 \%$ de la varianza total, siendo el contenido de flavonoides (PC1) el factor principal, que explicó el 66\%. La composición fenólica ha sido propuesta como herramienta de control de calidad respecto a estados del desarrollo para algunas especies de plantas, como
Rosmarinus oficinales (Del Baño et al., 2003) y Physalis angulata (CobaledaVelasco et al., 2017). La discriminación de los frutos de tomatillo sometidos a condiciones diferentes de salinidad, basada en los contenidos de flavonoides y antocianinas, y en el potencial inhibidor de $\alpha$-glucosidasa, obtenidos en el presente trabajo, indica que esos parámetros representan una herramienta de tipificación y de calidad con relación a las condiciones de crecimiento para los frutos de $P$. ixocarpa. 


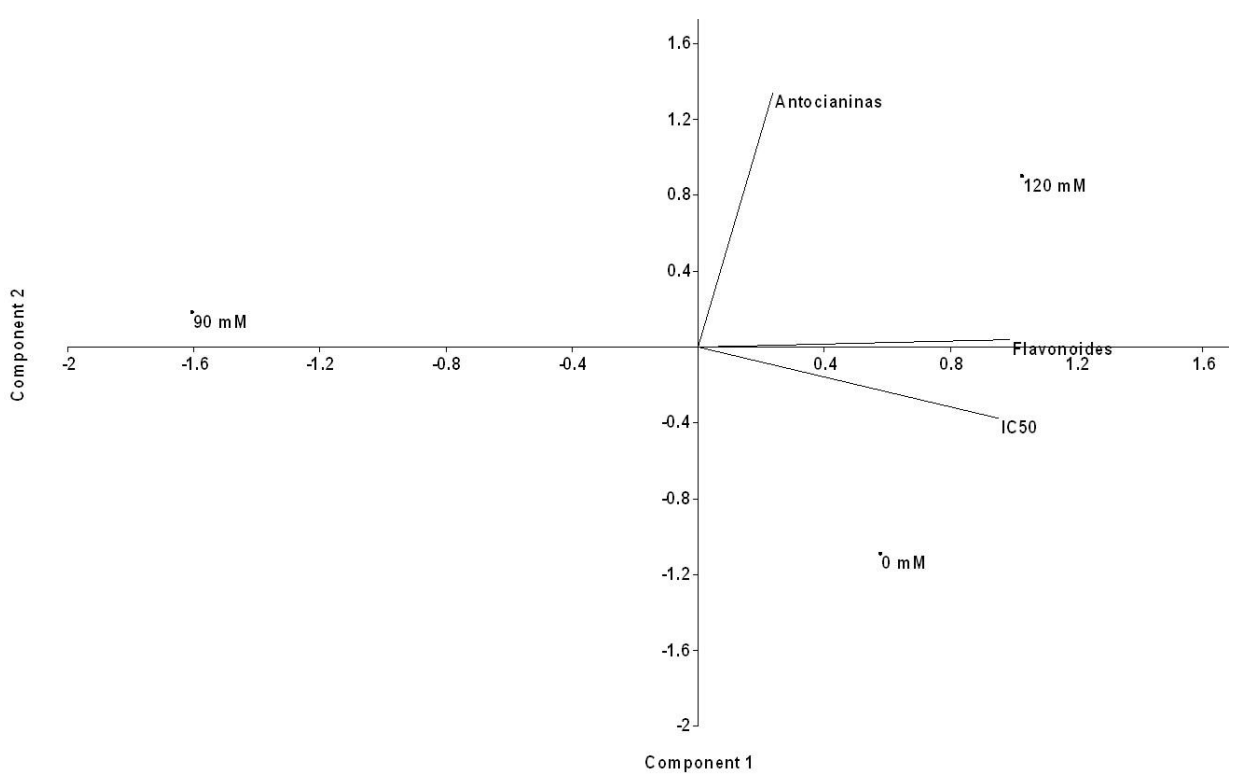

Figura 1. Resultado de un análisis de componentes principales, basado en el contenido de flavonoides, de antocianinas, y de $\mathrm{IC}_{50}$ de frutos de tomatillo (Physalis ixocapa) de plantas crecidas a diferentes concentraciones de $\mathrm{NaCl}(0,90$, o $120 \mathrm{mM})$.

\section{Conclusiones}

Los resultados obtenidos en el presente trabajo, permitieron evidenciar el incremento en el contenido de flavonoides y antocianinas de plantas sometidas a crecimiento en una concentración considerable de sal (120 $\mathrm{mM})$. De la misma manera se obtuvo mayor potencial inhibidor de la enzima $\alpha$ glucosidasa. Esta información podría utilizarse para la producción de sustancias que coadyuven en el tratamiento de enfermedades como la diabetes, potenciando el efecto de tratamientos que involucran también otras enfermedades.

\section{Literatura citada}

Asensi-Fabado, M-A., A. Amtmann y G. Perrella. (2017). Plant responses to abiotic stress: The chromatin context of transcriptional regulation. Biochimica et Biophysica Acta, 1860(1), 106-
122.

https://doi.org/10.1016/j.bbagrm.2 016.07.015

Asgar, M. A. (2013). Anti-diabetic potential of phenolic compounds: A Review. International Journal of Food Properties, 16(1), 91-103. https://doi.org/10.1080/10942912. 2011.595864

Choi, J. K., G. Murillo, B. N. Su, J. M. Pezzuloto, A. D. Kinghorn, R. G. Mehta. (2006). Ixocarpalactone A isolated from the Mexican tomatillo shows potent antiproliferative and apoptotic activity in colon cancer cells. The FEBS Journal, 273(24), 57145723.

https://doi.org/10.1111/j.17424658.2006.05560.x

Chutipaijit, S., S. Cha-um, K. Sompornpailin. (2009). Differential accumulations of proline and flavonoids in indica rice varieties against salinity. 
Pakistan Journal of Botany, 41(5), 2497-2506.

Cobaleda-Velasco, M., R. E AlanisBañuelos, N. Almaraz-Abarca, M. Rojas-López, L. S. GonzálezValdez, J. A. Ávila-Reyes, S. Rodrigo. (2017). Phenolic profiles and antioxidant properties of Physalis angulata L. as quality indicators. Journal of Pharmacy \& Pharmacognosy Research, 5(2), 114-128.

Del Baño, M. J., J. Lorente, J. Castillo, O. Benavente-García, J. A. Del Río, A. Ortuño, K. W. Quirin, D. Gerard. (2003). Phenolic diterpenes, flavones, and rosmarinic acid distribution during the development of leaves, flowers stems, and roots of Rosmarinus officinalis. Antioxidant activity. Journal of Agricultural and Food Chemistry, 51(15), 4227-4253. https://doi.org/10.1021/jf0300745

García-Luna P. P., G. López-Gallardo. (2007). Evaluación de absorción y metabolismo intestinal. Nutrición Hospitalaria, 22(2), 5-13.

Giusti, M. M., R. E. Wrolstad. (2001). Characterization and measurement of anthocyanins by UV-visible spectroscopy. Currents Protocols in Food Analytical Chemistry, OO(1), F1.2.1-F1.2.13. http://doi.org/10.1002/047114291 3.faf0102s00

González-Mendoza, D., O. GrimaldoJuárez, R. Soto-Ortiz, F. Escoboza-García, J. F. S. Hernández. (2010). Evaluation of total phenolics, anthocyanins and antioxidant capacity in purple tomatillo (Physalys ixocarpa) genotypes. African Journal of Biotechnology, 9(32), 5173-5176.
Hadjzadeh, M. A., Z. Rajaei, R. Moradi, A. Ghorbani. (2015). Effects of hydroalcoholic extract of watercress (Nasturtium officinale) leaves on serum glucose and lipid levels in diabetic rats. Indian Journal of Physiology and Pharmacology, 59(2), 223-230.

Hassan, H. A., H. M. Serag, M. S. Qadir, M. F. Ramadan. (2017). Cape gooseberry (Physalis peruviana) juice as modulator agent for hepatocellular carcinoma-linked apoptosis and cell cycle arrest. Biomedicine and Pharmacotherapy, 94, 1129-1137. http://dx.doi.org/10.1016/j.biopha. 2017.08.014

Henrich, M., B. F. Haller, M. Leonti. (2014). A perspective on natural products research and ethnopharmacology in Mexico: The Eagle and the Serpents on the Prickly Pear Cactus. Journal of Natural Products, 77(3), 678-689. http://dx.doi.org/10.1021/np40099 27 |

Kim, J-S., Y-S. Kwon, W-J. Chun, T-Y. Kim, J. Sun, C-Y-Yu, M-J. Kim. (2010). Rhus verniciflua Stokes flavonoid extracts have antioxidant, anti-microbial and $\alpha$ glucosidase inhibitory effect. Food Chemistry, 120(2), 539-543. http://dx.doi.org/10.1016/j.foodch em.2009.10.051

Kim, J-S., T. K. Hyun, M-J. Kim. (2011). The inhibitory effects of ethanol extracts from sorghum, foxtail millet and proso millet on $\alpha$ glucosidase and $\alpha$-amylase activities. Food Chemistry, 124(4), 1647-1651. https://doi.org/10.1016/j.foodche m.2010.08.020

Martínez-Lüscher, J., N. Torres, G. Hilbert, T. Richard, M. Sánchez- 
Díaz, S. Delrot, J. Aguirreolea, I. Pascual, E. Gomès. (2014). Ultraviolet-B radiation modifies the quantitative and qualitative profile of flavonoids and amino acids in grape berries. Photochemistry, 102, 106-114. http://dx.doi.org/10.1016/j.phytoc hem.2014.03.014

Minh, L. T., D. T. Khang, P. T. T. Ha, P. T. Tuyen, T. N. Minh, N. V. Quan, T. D. Xuan. (2016). Effects of salinity stress on growth and phenolics of rice (Oryza sativa L.). International Letters of Natural Sciences, 57, 1-10. http://dx.doi.org/10.18052/www.s cipress.com/ILNS.57.1

Ordoñez, A. A. L., J. D. Gomez, M. A. Vattuone, M. I. Isla. (2006). Antioxidant activities of Sechium edule (Jacq.) Swartz extracts. Food Chemistry, 97(3), 452-458. http://dx.doi.org/10.1016/j.foodch em.2005.05.024

Ramírez, F., G. Fisher, T. Davenport, J. Pinzón, C. Ulrichs. (2013). Cape gooseberrt (Physalis peruviana L.) phenology according to the $\mathrm{BBCH}$ phenological scale. Scientia Horticulturae, 162, 3942. http://dx.doi.org/10.1016/j.scienta. 2013.07.033

Sun L., M. Miao. (2020). Dietary polyphenols modulate starch digestion and glycaemic level: a review. Critical Reviews in Food Science and Nutrition, 60(4), 541555. http://dx.doi.org/10.1080/1040839 8.2018.1544883

Telesiński, A., J. Nowak, B. Smolik, A. Dubowska, N. Skrzypiec. (2008). Effect of soil salinity on activity of antioxidant enzymes and content of ascorbic acid and phenols in bean (Phaseolus vulgaris L.) plants. Journal of Elementology, 13(3), 401-409.

Vinayagam, R., M. Jayachandran, B. Xu. (2016). Antidiabetic effects of simple phenolic acids: a comprehensive review. Phytotherapy Research, 30(2), 184-199.

Walia, H., C. Wilson, P. Condamine, X. Liu, A. M. Ismail, L. Zeng, S. L. Wanamaker, J. Mandal, J. Xu, X. Cui, T. J. Close. (2005). Comparative transcriptional profiling of two contrasting rice genotypes under salinity stress during the vegetative growth stage. Plant Physiology, 139(2), 822-835.

www.plantphysiol.org/cgi/doi/10. 1104/pp.105.065961

Xu, Y. M., E. M. K. Wijeratne, A. L. Babyak, H. R. Marks, A. A. Brooks, P. Tewary, L. J. Xuan, W. Q. Wang, T. J. Sayers, A. A. Gunatilaka. (2017). Withanolides from aeroponically grown Physalis peruviana and their selective cytotoxicity to prostate cancer and renal carcinoma cells. Journal of Natural Products, 80(7), 1981-1991. http://dx.doi.org/10.1021/acs.jnatp rod.6b01129 